\title{
Contactless nonlinear optics mediated by long-range Rydberg interactions
}

\author{
Hannes Busche ${ }^{\dagger}$, Paul Huillery ${ }^{\dagger}$, Simon W. Ball, Teodora llieva, Matthew P. A. Jones \\ and Charles S. Adams *
}

In conventional nonlinear optics, linear quantum optics ${ }^{1,2}$, and cavity quantum electrodynamics ${ }^{3,4}$ to create effective photonphoton interactions photons must have, at one time, interacted with matter inside a common medium. In contrast, in Rydberg quantum optics ${ }^{5-10}$, optical photons are coherently and reversibly mapped onto collective atomic Rydberg excitations ${ }^{11}$, giving rise to dipole-mediated effective photon-photon interactions that are long range ${ }^{12,13}$. Consequently, a spatial overlap between the light modes is no longer required. We demonstrate such a contactless coupling between photons stored as collective Rydberg excitations in spatially separate optical media. The potential induced by each photon modifies the retrieval mode of its neighbour ${ }^{7,9,14,15}$, leading to correlations between them. We measure these correlations as a function of interaction strength, distance and storage time, demonstrating an effective interaction between photons separated by 15 times their wavelength. Contactless effective photon-photon interactions ${ }^{16}$ are relevant for scalable multichannel photonic devices ${ }^{15,17}$ and the study of strongly correlated many-body dynamics using light ${ }^{18}$.

In vacuum, photon-photon interactions are so weak that they are discernible only at cosmological scales ${ }^{19}$. This is a remarkable asset for astronomy, imaging and communications, but a serious obstacle for all-optical processing. In linear quantum optics, one can engineer an effective interaction via measurement ${ }^{1}$ which enables entanglement swapping between remote photons ${ }^{2}$; however, this relies on probabilistic post-selective measurements. Inside a medium, interactions between light fields are possible if the lightmatter coupling is nonlinear. For sufficiently strong nonlinearities, such as in cavity quantum electrodynamics (cQED) ${ }^{3}$ or Rydberg quantum optics ${ }^{5}$, one enters a quantum nonlinear regime ${ }^{20}$, allowing deterministic effective interactions at the single-photon level ${ }^{4,7-10}$. This has paved the way towards prototypical single-photon transistors $^{21,22}$, phase-shifters ${ }^{23}$, and photon gates ${ }^{24}$.

A unique feature of Rydberg-mediated quantum nonlinear optics $^{5}$ is that the interaction is long range ${ }^{12,13}$, and can be mediated through free space by virtual microwave photons. This adds new geometric degrees of freedom to the nonlinear optics tool box which could facilitate scalable photonic networks, quantum simulation with photons, and circumvent 'no-go' theorems that limit the scope for all-optical quantum information processing (QIP) $)^{15-17,25}$. To directly demonstrate the long-range character of Rydberg-mediated nonlinear optics, we realize two strongly interacting photonic channels in independent optical media separated by an adjustable distance $d$ of more than $10 \mu \mathrm{m}$. While stored as collective Rydberg excitations, van der Waals (vdW) interactions imprint non-uniform phase shifts ${ }^{14,15}$ that lead to reduced retrieval in the photons' initial modes (Fig. 1). By counting photons in the unperturbed modes, the reduction is manifest as a spatial anti-correlation in the observed photon statistics. The media can be viewed as two 'black-boxes' which make photons interact at a distance, suppressing simultaneous retrieval in both channels.

Consider two weak optical pulses focused into independent atomic media, labelled $A$ and $B$, and stored as strongly interacting collective Rydberg excitations or polaritons $s^{7,9}$ using electromagnetically induced transparency (EIT, see Fig. 2a) ${ }^{26}$. During storage, a photon in channel $A(B)$ is coherently converted into the collective atomic excitation

$$
|R\rangle_{A(B)}=\frac{1}{\sqrt{\mathcal{N}_{A(B)}}} \sum_{j \in A(B)} \mathrm{e}^{i \mathbf{k}_{A(B)} \cdot \mathbf{r}_{j}}\left|R_{j}\right\rangle_{A(B)}
$$

where $\mathcal{N}_{A(B)}$ is the atom number in the channel, $\mathbf{k}_{A(B)}$ the wavevector of the EIT light fields, $\mathbf{r}_{j}$ the position of atom $j$, and $\left|R_{j}\right\rangle_{A(B)}$ the collective state where atom $j$ is in Rydberg state $|r\rangle$ with all others in ground state $|g\rangle$. If the individual phases $\mathbf{k}_{A(B)} \cdot \mathbf{r}_{j(k)}$ in the collective excitation remain unchanged during storage, the phase and spatial mode of the photon are preserved. However, when photons are stored in both channels for a storage time $t_{\mathrm{st}}$, the two-channel collective state $|R\rangle_{A B}=|R\rangle_{A} \otimes|R\rangle_{B}$ evolves according to

$$
\hat{U}\left(t_{\mathrm{st}}\right)_{A B}=\sum_{j \in A, k \in B} \mathrm{e}^{-i V_{j k} t_{\mathrm{st}}}\left(\left|R_{j}\right\rangle_{A} \otimes\left|R_{k}\right\rangle_{B}\right)\left(\left\langle\left.R_{j}\right|_{A} \otimes\left\langle\left. R_{k}\right|_{B}\right)\right.\right.
$$

Here, $V_{j k}=n^{11} C_{6}^{\prime} / r_{j k}^{6}$ is the vdW potential, $n$ the principal quantum number of $|r\rangle, C_{6}^{\prime}$ the reduced vdW coefficient, and $r_{j k}=\left|\mathbf{r}_{j}-\mathbf{r}_{k}\right|$. The spatially dependent interaction-induced phase $V_{j k} t_{\mathrm{st}}$ accumulated between atom $j$ in $|R\rangle_{A}$ and $k$ in $|R\rangle_{B}$ leads to photon retrieval in different modes than before storage.

In the experiment (see Fig. 2) we prepare two ensembles of ${ }^{87} \mathrm{Rb}$ atoms in side-by-side optical tweezers separated by a distance $d=10-15 \mu \mathrm{m}$. We focus separate signal pulses, duration $350 \mathrm{~ns}$ and mean photon number $\approx 2.2$, into each ensemble with $1 / \mathrm{e}^{2}$ radii of approximately $1 \mu \mathrm{m}$. The signal photons are resonant with the $|g\rangle=\left|5 S_{1 / 2}, F=2, m_{F}=2\right\rangle \rightarrow|e\rangle=\left|5 P_{3 / 2}, F^{\prime}=3, m_{F}^{\prime}=3\right\rangle$ transition, as shown in Fig. 2a. Here, $F$ and $m F$ are the total angular momentum of the atomic state and its projection on the quantization axis, respectively, in units of $\hbar$; $S$ and $P$ denote the orbital electric angular momentum of 0 and 1 , and the subscripts specify the total electronic angular momentum (orbital plus spin), all in units of $\hbar$. A counter-propagating control field resonantly couples $|e\rangle \rightarrow|r\rangle=\left|n S_{1 / 2}\right\rangle$. Signal and control light have opposing circular polarization. To store signal photons, the control field is turned off, as indicated by the fading blue line in Fig. $2 \mathrm{~b}$. 


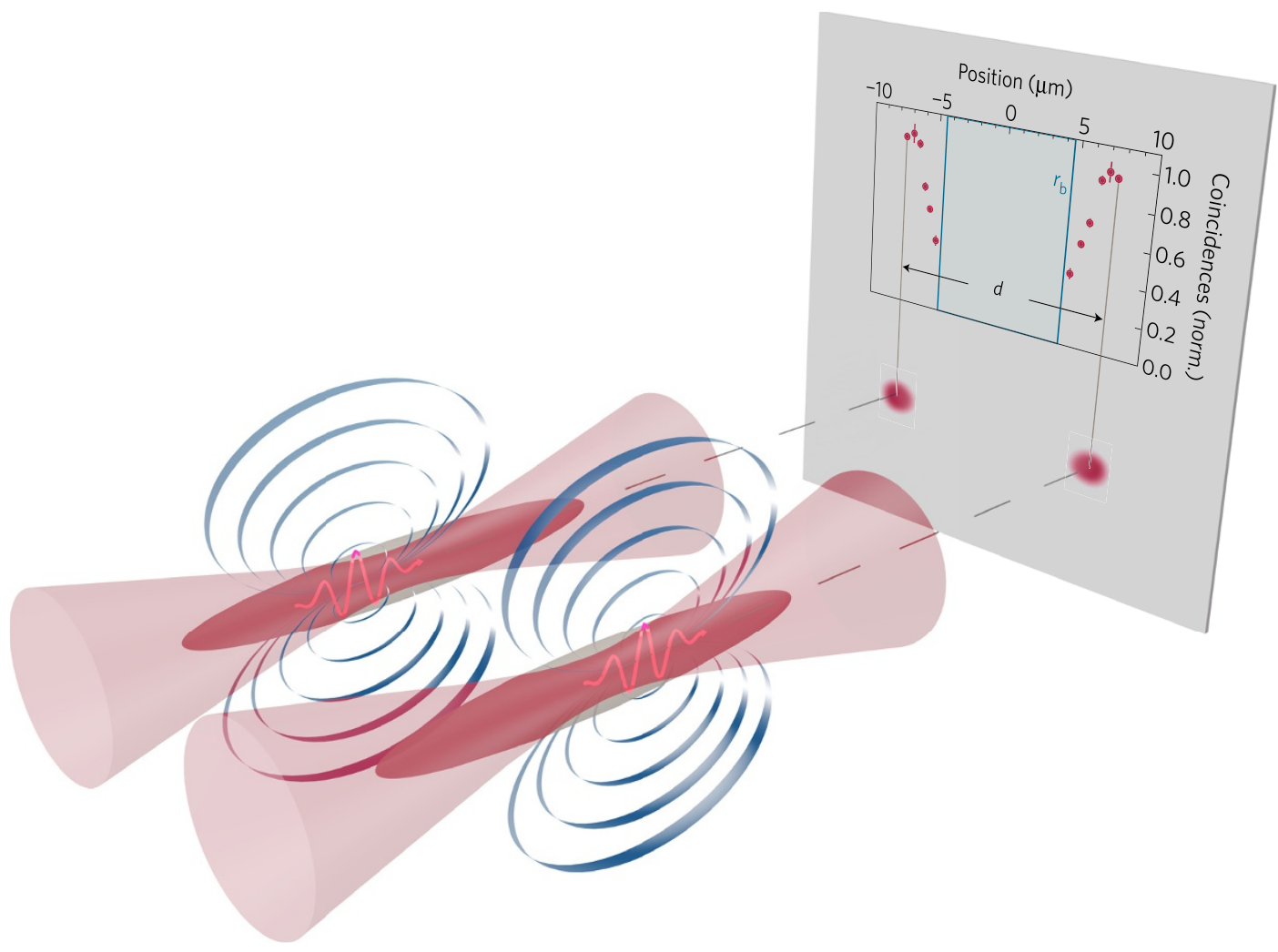

Figure 1 | Contactless interactions between photons stored as collective Rydberg excitations in spatially separated channels. Each channel consists of an optical mode tightly focused inside a microscopic cloud of ultra-cold atoms where signal photons are stored and retrieved. When two photons are stored in both channels simultaneously, vdW-interactions induce spatially dependent phase shifts which modify the retrieval modes. The reduction in the coincidence count rate as the two channels are moved closer together is shown on the screen. Strong cross-channel interaction effects appear at a separation $d=12 \mu \mathrm{m}$, which is both larger than the blockade radius $r_{b}$ for individual Rydberg atoms at $\left|80 S_{1 / 2}\right\rangle$ and equal to 15 times the optical wavelength.

a

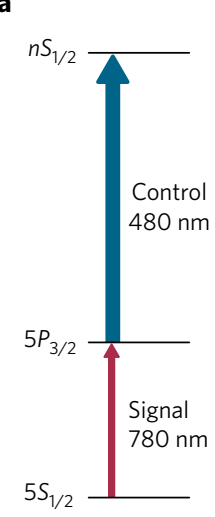

b

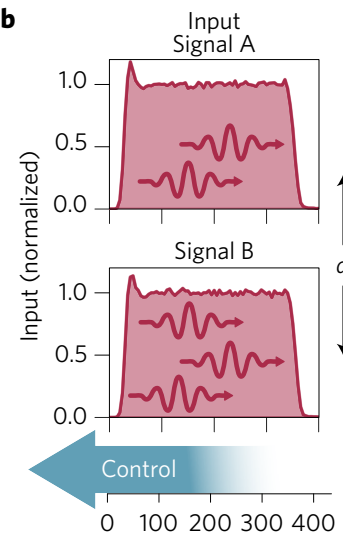

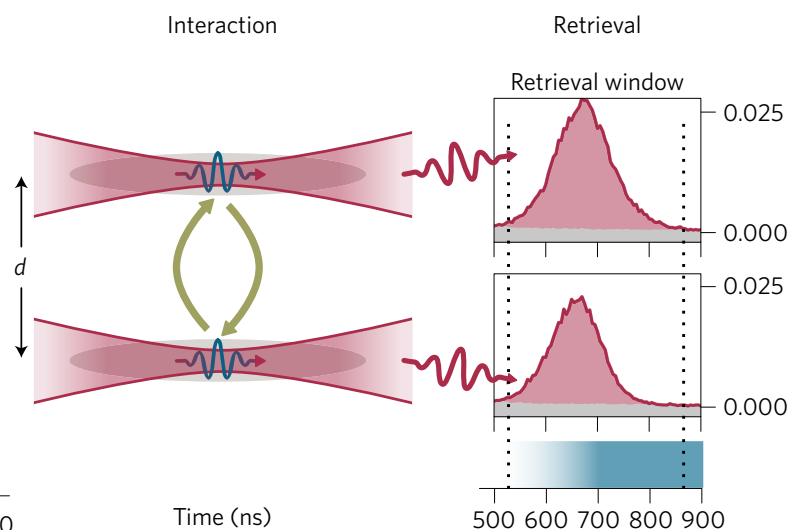

Detection

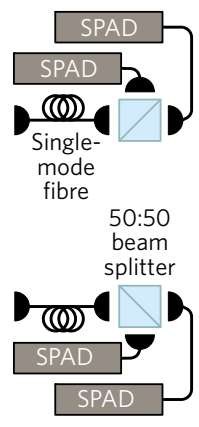

Figure 2 | Experimental realization. a, Transitions in ${ }^{87} \mathrm{Rb}$ used for ElT-based photon storage. Signal photons: $|g\rangle=\left|5 S_{1 / 2}, F=2, m_{F}=2\right\rangle \rightarrow$ $|e\rangle=\left|5 P_{3 / 2}, F^{\prime}=3, m_{F}^{\prime}=3\right\rangle$, control field: $|e\rangle \rightarrow|r\rangle=\left|n S_{1 / 2}\right\rangle$. b. Photon storage procedure. While signal pulses propagate through the media, the common control field is switched off. For retrieval, it is restored to its original value. The counting statistics of the retrieved signal photons are analysed using SPADs in two HBT set-ups. The actual input and retrieved signal pulses are shown. The cross-talk is zero as no retrieved photons above dark count (grey) are detected in channel $B$ when only storage medium $A$ is present, and vice versa.

Subsequently, we retrieve the signal photons by restoring the control field to its original intensity after a time $t_{\mathrm{st}} \approx 170 \mathrm{~ns}$. The photons retrieved in each channel are detected independently by pairs of single-photon detectors (SPADs) arranged in HanburyBrown-Twiss (HBT) set-ups. We verify that there is no cross-talk between channels in the storage-retrieval cycle by observing that no photon is retrieved in channel $A$ if only the medium of channel $B$ is present, and vice versa (Fig. 2b).
We analyse the counting statistics of photons for each channel using the correlation function

$$
g_{A}^{(2)}=\frac{\left\langle N_{A}\left(N_{A}-1\right)\right\rangle}{\left\langle N_{A}\right\rangle^{2}}
$$

where $N_{A}$ is the number of photons retrieved in channel $A$, and similarly for $g_{B}^{(2)}$. For principal quantum number $n=80$, 


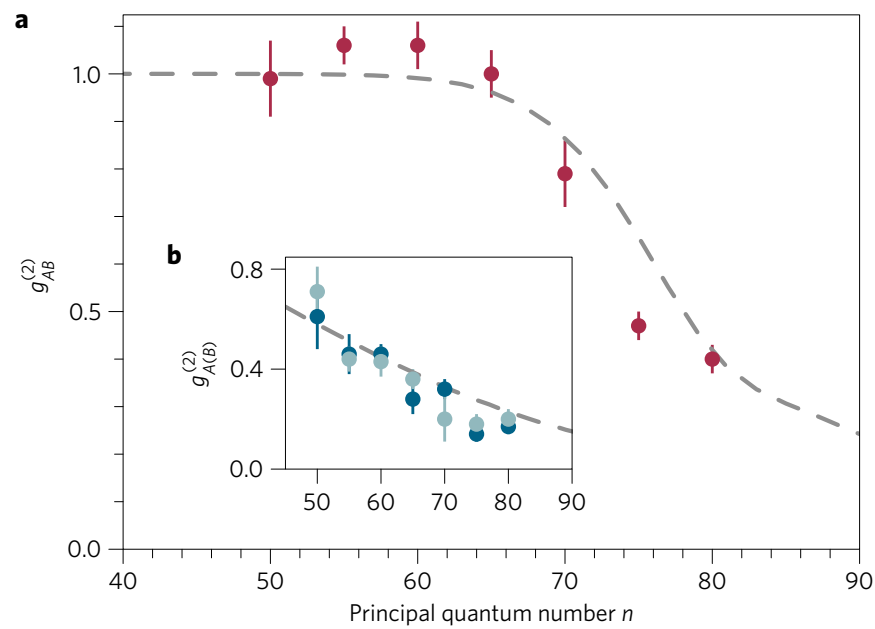

Figure 3 | Effect of interaction strength on retrieved photon statistics for channel separation $d=10 \mu \mathrm{m}$ and $t_{\mathrm{st}}=170 \mathrm{~ns}$. a, Cross-channel correlation function $g_{A B}^{(2)}$ measured for photon retrieval after storage in Rydberg states with principal quantum number $n$. As the interaction strength increases as $n^{11}$, an interaction between channels $g_{A B}^{(2)}<1$ is observed only for $n>65$. b. Single-channel correlation functions $g_{A(B)}^{(2)}$ in the absence of the other channel ( $A$ : light, $B$ : dark blue). The grey dashed lines are the expected results based on simulations (see text). Error bars correspond to statistical standard errors.

interaction-induced dephasing ${ }^{7,9,14}$ and Rydberg blockade ${ }^{27}$ limit the number of photons stored to close to one per channel, as indicated by strong anti-bunching in the single-channel correlation functions, $g_{A}^{(2)}=0.17 \pm 0.03$ and $g_{B}^{(2)}=0.20 \pm 0.04$. Consequently, the interactions between channels presented below occur at the level of individual photons.

To observe a cross-channel interaction, we store photons simultaneously in adjacent channels and analyse the cross-channel correlation function

$$
g_{A B}^{(2)}=\frac{\left\langle N_{A} N_{B}\right\rangle}{\left\langle N_{A}\right\rangle\left\langle N_{B}\right\rangle}
$$

For uncorrelated retrieval one expects $g_{A B}^{(2)}=1$, whereas $g_{A B}^{(2)} \neq 1$ would indicate an interaction between channels. For $d=10 \mu \mathrm{m}$ and $n=80$, we find $g_{A B}^{(2)}=0.40 \pm 0.03$ indicating an effective long-range interaction between non-overlapping, spatially separate photons.

To verify that the observed interactions arise from the vdW potential $V_{j k}$, we vary the interaction strength by changing the principal quantum number $n$. The data for fixed distance $d=10 \mu \mathrm{m}$ and $n$ between 50 and 80 (Fig. 3a) show that the interaction between channels increases with $n$, as expected. Interactions within each channel (Fig. 3b) also limit the number of photons stored and retrieved. For $n<65$, we observe no correlations between channels, but intra-channel interactions are still present.

To demonstrate the scaling of the Rydberg-mediated interaction with distance, we measure $g_{A B}^{(2)}$ as a function of the channel separation $d$, for a Rydberg state with $n=80$ (Fig. 4a). As $d$ is decreased, one alters the distribution of pair spacings, $r_{j k}$ ( $r_{j k} \gtrsim d$ for $j, k$ in adjacent channels) to smaller values, leading to stronger interactions. Consequently we observe a change from no correlation, $g_{A B}^{(2)}=1$, at $d=15 \mu \mathrm{m}$ to strong interaction-induced anti-correlations at $d=10 \mu \mathrm{m}$. As expected, the single-channel correlation functions are independent of $d$ (Fig. 4b). A key implication is the ability to separately tune the on-site and between-site interactions. This is equivalent to a decoupling of self- and crossKerr nonlinearities ${ }^{16}$ which is not possible in conventional nonlinear media and has the potential to become a major resource for nonlinear optics, for example, in optical QIP ${ }^{15,17,25}$.

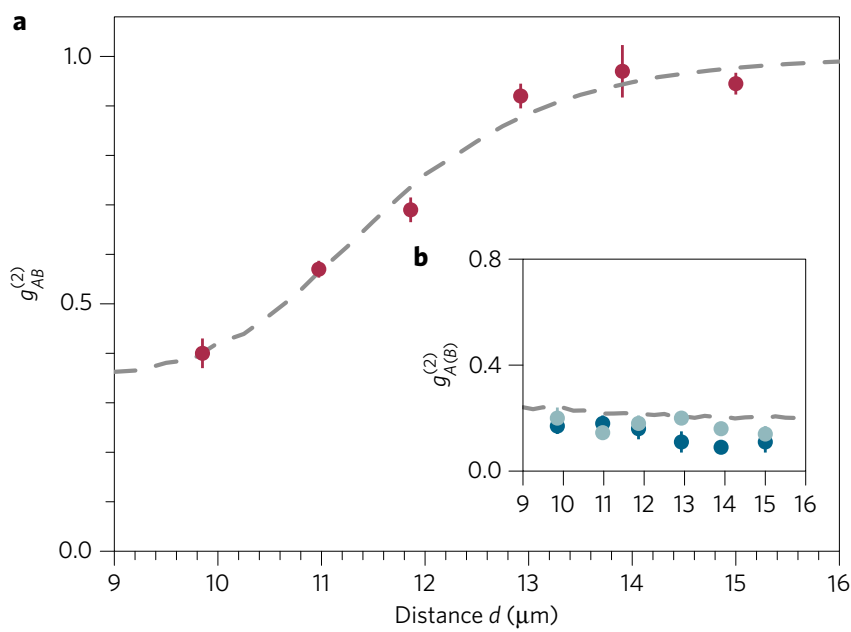

Figure 4 | Effect of channel separation $d$ on retrieved photon statistics for principal quantum number $n=80$ and $t_{s t}=170$ ns. a, Cross-channel correlation function $g_{A B}^{(2)}$ measured for photon retrieval after storage at different channel separations $d$. $\mathbf{b}$, Single-channel correlation functions $g_{A(B)}^{(2)}$ in the absence of the other channel ( $A$ : light, $B$ : dark blue). The change in $g_{A B}^{(2)}$ while the single-channel values $g_{A(B)}^{(2)}$ remain approximately constant as expected, shows that inter-channel interactions can be independently tuned by adjusting $d$. The grey dashed lines show simulation results. Error bars correspond to statistical standard errors.

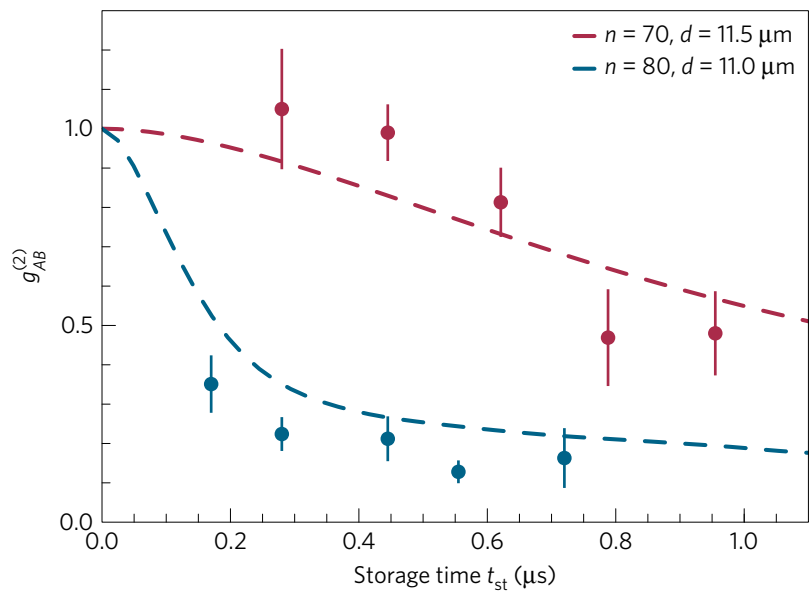

Figure 5 | Effect of the storage time $t_{\mathrm{st}}$ on retrieved photon statistics. Cross-channel correlation function $g_{A B}^{(2)}$ measured for photon retrieval after storage for a time $t_{\mathrm{st}}$ for principal quantum number $n=70$ and 80 and channel separation $d=11.5 \mu \mathrm{m}$ and $11.0 \mu \mathrm{m}$, respectively. The dashed lines show simulation results. Error bars correspond to statistical standard errors. The maximum storage time is limited by the finite temperature of the atomic ensemble which leads to motional dephasing. Note that the evaporative cooling stage (Methods) employed in all other experiments is omitted, resulting in slightly larger atomic media and the observation of cross-talk in signal absorption at $d=11.5 \mu \mathrm{m}$. No cross-talk is observed in photon storage.

To compare our results to theory, we extend previous theoretical work ${ }^{14}$ on interaction-induced dephasing within a single channel to model the effect of phase shifts in our dual-channel geometry (dashed lines in Figs 3-5, see Methods for more details). Spatial averaging of the phase shifts over all atoms contributing to the collective states means that interaction falls off more slowly than for the individual pair-wise terms, $V_{j k}$. We also verified that anticorrelations increase with the storage time $t_{\text {st }}$ (Fig. 5) as expected, because the interaction-induced phase scales as $V_{j k} t_{\mathrm{st}}$. This and the 
good agreement between theory and experiment when we vary the interaction strength (Fig. 3) or distance (Fig. 4) indicates that interaction-induced phase shifts make an important contribution to the overall interaction. Although the channels are separated by more than the radius, $r_{\mathrm{b}}\left(80 S_{1 / 2}\right) \approx 9 \mu \mathrm{m}<d$, for inter-channel dipole blockade between collective excitations ${ }^{11,27}$, blockade effects beyond $d$ associated with the storage protocol ${ }^{18}$ may also contribute to the interaction.

Rydberg-mediated long-range interactions between spatially separated optical photons are a novel and powerful addition to optics. If tailored to imprint uniform phase shifts, the micron-scale interaction length provides a convenient starting point for scalable all-optical QIP ${ }^{15,17}$ based on tweezer arrays ${ }^{28}$ or coupled to optical circuits in integrated photonic waveguides ${ }^{29}$. Quantum simulators based on dipolar interactions, for example, of spin models ${ }^{30}$ or nonradiative energy transfer ${ }^{31}$, may now be realized with photons stored as collective Rydberg excitations, providing the added benefit of fast coherent read-out and the ability to measure both amplitude and phase.

\section{Methods}

Methods, including statements of data availability and any associated accession codes and references, are available in the online version of this paper.

\section{Received 19 December 2016; accepted 7 February 2017;} published online 6 March 2017

\section{References}

1. Knill, E., Laflamme, R. \& Milburn, G. J. A scheme for efficient quantum computation with linear optics. Nature 409, 46-52 (2001).

2. Pan, J.-W., Bouwmeester, D., Weinfurter, H. \& Zeilinger, A. Experimental entanglement swapping: entangling photons that never interacted. Phys. Rev. Lett. 80, 3891-3894 (1998).

3. Reiserer, A. \& Rempe, G. Cavity-based quantum networks with single atoms and optical photons. Rev. Mod. Phys. 87, 1379-1418 (2015).

4. Birnbaum, K. M. et al. Photon blockade in an optical cavity with one trapped atom. Nature 436, 87-90 (2005).

5. Firstenberg, O., Adams, C. S. \& Hofferberth, S. Nonlinear quantum optics mediated by Rydberg interactions. J. Phys. B 49, 152003 (2016).

6. Pritchard, J. D. et al. Cooperative atom-light interaction in a blockaded Rydberg ensemble. Phys. Rev. Lett. 105, 193603 (2010)

7. Dudin, Y. O. \& Kuzmich, A. Strongly interacting Rydberg excitations of a cold atomic gas. Science 336, 887-889 (2012).

8. Peyronel, T. et al. Quantum nonlinear optics with single photons enabled by strongly interacting atoms. Nature 488, 57-60 (2012).

9. Maxwell, D. et al. Storage and control of optical photons using Rydberg polaritons. Phys. Rev. Lett. 110, 103001 (2013).

10. Firstenberg, O. et al. Attractive photons in a quantum nonlinear medium. Nature 502, 71-75 (2013)

11. Ebert, M., Kwon, M., Walker, T. G. \& Saffman, M. Coherence and Rydberg blockade of atomic ensemble qubits. Phys. Rev. Lett. 115, 093601 (2015).

12. Gorshkov, A. V., Otterbach, J., Fleischhauer, M., Pohl, T. \& Lukin, M. D Photon-photon interactions via Rydberg blockade. Phys. Rev. Lett. 107, 133602 (2011)

13. Sevinçli, S., Henkel, N., Ates, C. \& Pohl, T. Nonlocal nonlinear optics in cold Rydberg gases. Phys. Rev. Lett. 107, 153001 (2011)
14. Bariani, F., Goldbart, P. M. \& Kennedy, T. A. B. Dephasing dynamics of Rydberg atom spin waves. Phys. Rev. A 86, 041802(R) (2012).

15. Khazali, M., Heshami, K. \& Simon, C. Photon-photon gate via the interaction between two collective Rydberg excitations. Phys. Rev. A 91, 030301(R) (2015).

16. He, B., Sharypov, A. V., Sheng, J., Simon, C. \& Xiao, M. Two-photon dynamics in coherent Rydberg atomic ensemble. Phys. Rev. Lett. 112, 133606 (2014).

17. Paredes-Barato, D. \& Adams, C. S. All-optical quantum information processing using Rydberg gates. Phys. Rev. Lett. 112, 040501 (2014).

18. Moos, M., Höning, M., Unanyan, R. \& Fleischhauer, M. Many-body physics of Rydberg dark-state polaritons in the strongly interacting regime. Phys. Rev. A 92, 053846 (2015)

19. Heisenberg, W. \& Euler, H. Folgerungen aus der Diracschen Theorie des Positrons. Z. Phys. 98, 714-732 (1936).

20. Chang, D. E., Vuletić, V. \& Lukin, M. D. Quantum nonlinear optics-photon by photon. Nat. Photon. 8, 685-694 (2014).

21. Gorniaczyk, H., Tresp, C., Schmidt, J., Fedder, H. \& Hofferberth, S. Single-photon transistor mediated by interstate Rydberg interactions. Phys. Rev. Lett. 113, 053601 (2014).

22. Tiarks, D., Baur, S., Schneider, K., Dürr, S. \& Rempe, G. Single-photon transistor using a Förster resonance. Phys. Rev. Lett. 113, 053602 (2014).

23. Tiarks, D., Schmidt, S., Rempe, G. \& Dürr, S. Optical $\pi$ phase shift created with a single-photon pulse. Sci. Adv. 2, 160036 (2016).

24. Hacker, B., Welte, S., Rempe, G. \& Ritter, S. A photon-photon quantum gate based on a single atom in an optical resonator. Nature 536, 193-196 (2016).

25. Shapiro, J. H. Single-photon Kerr nonlinearities do not help quantum computation. Phys. Rev. A 73, 062305 (2006).

26. Fleischhauer, M. \& Lukin, M. D. Dark-state polaritons in electromagnetically induced transparency. Phys. Rev. Lett. 84, 5094-5097 (2000).

27. Lukin, M. D. et al. Dipole blockade and quantum information processing in mesoscopic atomic ensembles. Phys. Rev. Lett. 87, 037901 (2001).

28. Nogrette, F. et al. Single-atom trapping in holographic $2 \mathrm{D}$ arrays of microtraps with arbitrary geometries. Phys. Rev. X 4, 021034 (2014).

29. Kohnen, M. et al. An array of integrated atom-photon junctions. Nat. Photon. 5, 35-38 (2011)

30. Barredo, D. et al. Coherent excitation transfer in a spin chain of three Rydberg atoms. Phys. Rev. Lett. 114, 113002 (2015).

31. Günter, G. et al. Observing the dynamics of dipole-mediated energy transport by interaction-enhanced imaging. Science 342, 954-956 (2013).

\section{Acknowledgements}

We thank D. Paredes-Barato, K. J. Weatherill, and especially D. J. Szwer for early contributions to the experimental set-up, and S. Hofferberth for suggestions on the manuscript. We acknowledge financial support from the EU's FP7 and Horizon 2020 programmes through the Marie Curie ITN 'COHERENCE' (FP7-PEOPLE-2010-ITN-265031), FET-PROACT project 'RySQ' (H2020-FETPROACT-2014-640378-RYSQ), and a Marie Skłodowska-Curie Fellowship to P.H. (H2020-MSCA-IF-2014-660028), EPSRC grant 'Rydberg soft matter' (EP/M014398/1), DSTL, as well as Durham University.

\section{Author contributions}

All authors contributed to the experiment, analysis and discussion of the results, as well as the preparation of the manuscript.

\section{Additional information}

Supplementary information is available in the online version of the paper. Reprints and permissions information is available online at www.nature.com/reprints. Publisher's note: Springer Nature remains neutral with regard to jurisdictional claims in published maps and institutional affiliations. Correspondence and requests for materials should be addressed to C.S.A.

\section{Competing financial interests}

The authors declare no competing financial interests. 


\section{Methods}

Experimental set-up and preparation of atomic media. Each photonic channel consists of a tightly focused signal beam and a microscopic optical tweezer confining cold atoms. This requires near diffraction limited optical resolution.

We achieve this using a pair of aspheric lenses (numerical aperture $\approx 0.5$, focal length $\approx 10 \mathrm{~mm}$ ) placed inside the ultrahigh vacuum chamber in which experiments take place. As Rydberg atoms are highly sensitive to electric fields, conductive transparent indium tin oxide (ITO) coatings have been applied to the dielectric lens surfaces facing the atoms to avoid charge build-up in their vicinity. A detailed description of the experimental set-up can be found elsewhere ${ }^{32}$.

The experimental procedure is as follows. Initially we trap atoms in a magneto-optical trap (MOT) which is loaded from a cold bright atomic beam created by a two-dimensional MOT in $100 \mathrm{~ms}$. Subsequently, we increase their density and decrease their temperature by first compressing the MOT via an increase in the magnetic field gradient and then subjecting them to a grey optical molasses phase. The red-detuned dipole trap beams at a wavelength of $910 \mathrm{~nm}$ are focused to $1 / \mathrm{e}^{2}$ radii of approximately $4.5 \mu \mathrm{m}$, creating traps with depths of approximately $400 \mu \mathrm{K}$, and are turned on right from the start of the MOT loading. Once the MOT is switched off, we apply free evaporative cooling for $400 \mathrm{~ms}$, which reduces the volume of the trapped atoms to avoid any overlap between signal beam $A$ and medium $B$, or vice versa. The resulting two cigar-shaped clouds with estimated radii (standard deviations of Gaussian atom distribution) approximately $20 \mu \mathrm{m}$ (axial) and $1.5 \mu \mathrm{m}$ (radial), contain on the order of $10^{3}$ atoms each. These estimates are based on upper bounds obtained from imaging and the calculated trapping potentials. They are consistent with our observation that the absorption is reduced if the signal beam waist exceeds $2 \mu \mathrm{m}$, and with the values obtained from fitting the dephasing model as described below.

The signal beams are overlapped with the respective trapping beams for each channel to simplify adjusting the distance $d$ between channels by enabling simultaneous alignment of both beams. The channel separation is measured and adjusted by imaging the signal foci at an approximately 39 -fold magnification. Besides verifying the absence of cross-talk in photon storage as shown in Fig. 2, we also verify that there is no significant cross-talk in the absorption of the signal light providing stronger evidence of no overlap between channels. Whereas we observe $2-3 \%$ cross-absorption for $d=10 \mu \mathrm{m}$, it vanishes for $d_{A B} \geq 11 \mu \mathrm{m}$ (see figure in Supplementary Information), well below the threshold where the photon retrieval becomes uncorrelated between channels as $g_{A B}^{(2)}$ approaches 1 (see Fig. $4 \mathrm{a}$ ).

Photon storage. Following the preparation of the atom clouds, we optically pump the atoms to the state $|g\rangle=\left|5 S_{1 / 2}, F=2, m_{F}=2\right\rangle$. Subsequently, the trapping light is turned off for $1.5 \mu$ s every $4.55 \mu$ s, during which we carry out one storage and retrieval sequence as shown in Fig. 2b. Since heating of the ensemble and atom loss are low due to the weak signal pulse, we can repeat this sequence 25,000 times before reloading the traps. This corresponds to an effective cycle rate of $33.5 \mathrm{kHz}$, mostly determined by the time needed to prepare the atomic ensembles-that is, loading of the MOT and evaporation. In total, we repeat the storage and retrieval sequence 25 million times for each datapoint presented-except for the data in Fig. 5, where up to 156 million repetitions are carried out due to lower retrieval at longer storage times. The initial Rabi frequency of the control field is

$\Omega_{\mathrm{C}} / 2 \pi \approx 9 \mathrm{MHz}$ for the data in Fig. 3 and $\Omega_{\mathrm{C}} / 2 \pi \approx 7 \mathrm{MHz}$ for figures 4 (except for $d=10 \mu \mathrm{m}$ where $\Omega_{\mathrm{C}} / 2 \pi \approx 9 \mathrm{MHz}$ ) and 5 . The storage times $t_{\mathrm{st}}$ excludes the fall and rise time of the control field.

The overall efficiencies for the photon storage/retrieval protocol in these experiments are typically between 1 and 3\%, limited by strong interactions within the same channel and the small cloud size and thus limited optical depth of the atomic media ${ }^{33}$. Working at weaker signal pulses with mean photon number well below 1 and using slightly larger atom clouds, we have previously achieved efficiencies on the order of approximately $10 \%$, while the current published record for storage in Rydberg states stands at approximately $20 \%$ (ref. 23). Increasing the optical depth by increasing the atomic density proves difficult, as additional dephasing occurs due to interactions between ground state atoms and Rydberg electrons if the inter-atomic spacing becomes equivalent to the size of the electron orbit $^{34}$, although this might not be problematic when engineering dissipative interactions ${ }^{33}$. Recently, ordered atomic monolayers have been suggested as a promising alternative to achieve high optical depth at well-controlled inter-atomic spacings ${ }^{35}$

Single-photon detection and correlation analysis. The signal light retrieved in the unperturbed modes is coupled into polarization-maintaining single-mode fibres to filter out stray light and spontaneous emission from the decay of dephased polaritons. The 50:50 beam splitters for HBT interferometry are located behind the fibres. We record all detection events independently for each detector using a home-built FPGA system with a timing resolution of $5 \mathrm{~ns}$. The detection efficiencies of signal photons emitted from the atomic ensembles are typically $20 \%$ for channel $A$ and $21 \%$ for channel $B$, including all loss on subsequent optical elements-that is, transmission loss on the ITO coating on the in-vacuum lens, laser line filters, and finite coupling efficiencies into the single-mode fibres, as well as the quantum efficiencies of the SPADs.

To evaluate the correlation functions given in the main text, we analyse the photon statistics in software post-processing based on the recorded timestamps of detection events. Due to the finite dead-time of the SPADs, we limit our analysis of photon numbers to as many detection events as detectors (that is, two per channel) within the retrieval window. We account for imbalances in the 50:50 HBT splitting ratio resulting from imperfect beam splitters and imbalanced detection efficiencies.

Formal description of the interaction-induced phase shift mechanism and simulations of retrieved photon statistics. In the following, we describe the mechanism that gives rise to the phase shift in detail and simulate the effect on the retrieved photon statistics. We build upon work by Bariani et al. ${ }^{14}$, who describe interaction-induced dephasing between collective Rydberg excitations within a single atomic ensemble. We expand their work to include multiple, arbitrarily shaped signal modes and atomic media to simulate the two-channel geometry of our experiment. We consider the following two scenarios: a single channel containing no more than two excitations in the same medium; and a pair of spatially separated channels containing no more than a single excitation per channel. The truncation to two excitations is justified since, in comparison, triple excitations are highly unlikely due to the low number of photons which are retrieved per storage cycle (on the order of a few 0.01). Despite its simplicity, this approach allows for full exploration of the mechanism and successfully reproduces experimental data.

Initially, we consider storage in the same channel (here labelled $A$ ). Starting from the atomic ensemble's collective ground state $|G\rangle_{A}$, storage of $v$ photons results in the collectively excited state $\left|R^{(v)}\right\rangle_{A}=\left(\hat{S}_{A}^{\dagger}\right)^{v}|G\rangle_{A} / \sqrt{\nu !}$ where

$$
\hat{S}_{A}^{\dagger}=\frac{1}{\sqrt{\sum_{j} \epsilon_{A}\left(\mathbf{r}_{j}\right)^{2}}} \sum_{j} \epsilon_{A}\left(\mathbf{r}_{j}\right) \mathrm{e}^{i \varphi_{A}\left(\mathbf{r}_{j}\right)}|r\rangle_{j, A}\left\langle\left. g\right|_{j, A}\right.
$$

describes the creation of an (additional) collective excitation. Here, $|g\rangle_{j, A}$ and $|r\rangle_{j, A}$ correspond to the ground and Rydberg states of atom $j, \epsilon_{A}\left(\mathbf{r}_{j}\right)$ and $\varphi_{A}\left(\mathbf{r}_{j}\right)$ are, respectively, the electric field amplitude of the signal mode and the combined phase of both EIT fields at the position $\mathbf{r}_{\mathbf{j}}$ of atom $j$. We preserve approximately correct normalization in the limit $v \ll \mathcal{N}_{A}$. We consider the atomic ensemble of a single channel to be in a superposition $|R\rangle_{A}=\sum_{v \leq 2} c_{v}\left|R^{(v)}\right\rangle_{A}$ following storage, where the coefficients $c_{v}$ describe the distribution between excitation numbers, neglecting more than two excitations as explained above.

In the presence of van der Waals interactions described by $V_{j k}=n^{11} C_{6}^{\prime} / r_{j k}^{6}$, the individual Rydberg excitation pairs contributing to $\left|R^{(2)}\right\rangle_{A}$ evolve during $t_{\mathrm{st}}$ according to

$$
\hat{U}_{(A)}\left(t_{\mathrm{st}}\right)=\sum_{j, k>j} \mathrm{e}^{-i V_{j k} t_{\mathrm{st}}}\left|R_{j, k}\right\rangle_{A}\left\langle\left. R_{j, k}\right|_{A}\right.
$$

where $\left|R_{j, k}\right\rangle_{A}$ corresponds to the collective state where atoms $j$ and $k$ are excited. Since the phase shifts $V_{j k} t_{\text {st }}$ acquired between pairs vary with position, the collective state dephases as a consequence of the interactions, thus reducing the probability to retrieve both photons in the original signal mode from $\hat{U}_{A}\left(t_{\mathrm{st}}\right)\left|R^{(2)}\right\rangle_{A}$. The overlap

$$
\begin{aligned}
D_{A} & =\frac{\left\langle\left. G\right|_{A} \hat{S}_{A}^{2} \hat{U}_{A}\left(t_{\mathrm{st}}\right)\left(\hat{S}_{A}^{\dagger}\right)^{2} \mid G\right\rangle_{A}}{\left\langle\left. G\right|_{A} \hat{S}_{A}^{2}\left(\hat{S}_{A}^{\dagger}\right)^{2} \mid G\right\rangle_{A}} \\
& =\frac{\sum_{j, k>j}\left(\epsilon_{A}\left(\mathbf{r}_{j}\right) \epsilon_{A}\left(\mathbf{r}_{k}\right)\right)^{2} \mathrm{e}^{-i V_{j k} t_{\mathrm{st}}}}{\sum_{j, k>j}\left(\epsilon_{A}\left(\mathbf{r}_{j}\right) \epsilon_{A}\left(\mathbf{r}_{k}\right)\right)^{2}}
\end{aligned}
$$

between $|G\rangle_{A}$ and the collective state of the ensemble after retrieval of two photons in the signal mode from the time-evolved doubly excited state quantifies the dephasing, and allows us to compare the experimentally measured correlation function $g_{A}^{(2)}$ to theory via

$$
g_{A}^{(2)}=\frac{2\left|D_{A}\right|^{2}\left|c_{2}\right|^{2}}{\left(\left|c_{1}\right|^{2}+2\left|D_{A}\right|^{2}\left|c_{2}\right|^{2}\right)^{2}}
$$

Building on the single-channel case, we quantify interactions between photons stored as collective Rydberg excitations in two adjacent, non-overlapping channels. For two channels $A$ and $B$, the initial state with exactly $v(\mu)$ Rydberg excitations in channels $A(B)$ after photon storage can be written as the product $\left|R^{(v, \mu)}\right\rangle_{A B}=\left|R^{(v)}\right\rangle_{A} \otimes\left|R^{(\mu)}\right\rangle_{B}$ of the individual single-channel states. Hence, the collective state of both channels immediately after storage is $|R\rangle_{A B}=\sum_{v, \mu \in\{0,1\}} c_{v, \mu}\left|R_{A B}^{(v, \mu)}\right\rangle$, where $c_{v, \mu}$ again arise from the photon number distributions of the original signal pulses. Evolving over $t_{\mathrm{st}}$, the individual excitation pairs in $\left|R^{(1,1)}\right\rangle_{A B}$ again acquire non-uniform interaction-induced phases as

$$
\hat{U}\left(t_{\mathrm{st}}\right)_{A B}=\sum_{j \in A, k \in B} \mathrm{e}^{-i V_{j k} t_{\mathrm{st}}}\left(\left|R_{\mathrm{j}}\right\rangle_{A} \otimes\left|R_{k}\right\rangle_{B}\right)\left(\left\langle\left.R_{j}\right|_{A} \otimes\left\langle\left. R_{k}\right|_{B}\right)\right.\right.
$$


To estimate the effect of the imprinted phase shifts on the retrieved photon statistics, we now consider the overlap

$$
D_{A B}=\frac{\left\langle\left. G\right|_{A B} \hat{S}_{A} \hat{S}_{B} \hat{U}_{A B}\left(t_{\mathrm{st}}\right) \hat{S}_{A}^{\dagger} \hat{S}_{B}^{\dagger} \mid G\right\rangle_{A B}}{\left\langle\left. G\right|_{A B} \hat{S}_{A} \hat{S}_{B} \hat{S}_{A}^{\dagger} \hat{S}_{B}^{\dagger} \mid G\right\rangle_{A B}}
$$

where $|G\rangle_{A B}=|G\rangle_{A} \otimes|G\rangle_{B}$ and obtain

$$
g_{A B}^{(2)}=\frac{\left|D_{A B}\right|^{2}\left|c_{1,1}\right|^{2}}{\left(\left|c_{1,0}\right|^{2}+\left|D_{A B}\right|^{2}\left|c_{1,1}\right|^{2}\right)\left(\left|c_{0,1}\right|^{2}+\left|D_{A B}\right|^{2}\left|c_{1,1}\right|^{2}\right)}
$$

To compare experimental results to theory, we calculate both cross- and single-channel correlation functions as above based on the experimental parameters described at the beginning of the Methods, assuming $\mathcal{N}_{A(B)}=1,000$ atoms per trap. The numbers of photons stored in each channel follow a Poissonian distribution with mean $\bar{\nu}, \bar{\mu}=0.01$, with no initial correlation between channels and neglecting blockade ${ }^{27}$ effects: $\left|c_{v}\right|^{2}=\mathrm{e}^{-\bar{v}} \bar{\nu}^{\nu} / \nu$ ! and $\left|c_{v, \mu}\right|^{2}=\left|c_{v}\right|^{2}\left|c_{\mu}\right|^{2}$. By varying both $\mathcal{N}_{A(B)}$ and $\bar{v}(\bar{\mu})$, we verify that their values do not impact the results provided that the condition $\bar{v}(\bar{\mu}) \ll 1 \ll \mathcal{N}_{A(B)}$ is met. The storage time is $t_{\mathrm{st}}=170 \mathrm{~ns}$ (except for simulations in Fig. 5) and the interaction strength of $V_{j k}$ is set based on the Rydberg state considered. The simulation results are averaged over ten different instances for the atom distributions per datapoint. Although the model does not include any free parameter itself, $t_{\mathrm{st}}$ and the atom cloud sizes are not precisely experimentally verifiable (see above), and we use them as adjustable parameters.

Data availability. The data presented in this letter are available at http://dx.doi.org/doi:10.15128/r1wh246s12j.

\section{References}

32. Busche, H., Ball, S. W. \& Huillery, P. A high repetition rate experimental setup for quantum non-linear optics with cold Rydberg atoms. Eur. Phys. J. Spec. Top. 225, 2839-2861 (2016).

33. Gorshkov, A. V., Nath, R. \& Pohl, T. Dissipative many-body quantum optics in Rydberg media. Phys. Rev. Lett. 110, 153601 (2013).

34. Gaj, A. et al. From molecular spectra to a density shift in dense Rydberg gases. Nat. Commun. 5, 4546 (2014).

35. Bettles, R. J., Gardiner, S. A. \& Adams, C. S. Enhanced optical cross section via collective coupling of atomic dipoles in a 2D array. Phys. Rev. Lett. 116, 103602 (2016) 